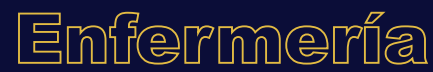

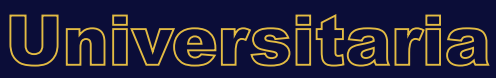

\section{Tecnologías en la enseñanza en enfermería, innovación y uso de TICs: revisión integrativa}

\author{
Innovation and use of information and \\ communication technologies in nursing \\ teaching: An integrative review
}

\section{Tecnologias no ensino em enfermagem, inovação e uso de TICs: revisão integrativa}

\section{A.L. Araújo-Girão ${ }^{a^{*}}$, M.L. Silva-Nunes Cavalcante ${ }^{b}$, I. Costa-Lima de Oliveirac ${ }^{c}$, S. Freitas-Aires ${ }^{\mathrm{d}}$, S.K. Paz-de Oliveira ${ }^{e}$, R.E. Fontenele-Lima de Carvalhof}

ORCID

a $\underline{0000-0003-2746-4801}$

d

b $0000-0003-4830-9413$

e $0000-0003-3902-8046$

c0000-0002-7460-9244

f $\underline{0000-0002-3406-9685}$

${ }^{1}$ Universidad Estatal de Ceará, Programa de Pos-Graduación Cuidados Clínicos en Enfermería y Salud, Fortaleza, Ceará, Brasil

Recibido: 14 enero 2020

Aceptado: 29 agosto 2020

\section{RESUMEN}

Introducción: La utilización de los recursos digitales modernos para la enseñanza constituye una estrategia para atender las nuevas necesidades de aprendizaje. En el ámbito de enfermería puede facilitar la inserción de los estudiantes en la práctica clínica en modo virtual para que desarrollen sus habilidades y competencias.

Objetivo: Analizar las evidencias sobre la utilización de tecnologías en la enseñanza de enfermería.

Métodos: Revisión integrativa realizada en el portal de revistas SciELO y en las bases de datos IBECS, LILACS, BDENF, Web of Science, PubMed y CINAHL.

*Autora para correspondencia. Correo electrónico: aliviagirao@gmail.com https://doi.org/10.22201/eneo.23958421e.2020.4.763

1665-7063/@ 2020 Universidad Nacional Autónoma de México, Escuela Nacional de Enfermería y Obstetricia. Este es un artículo Open Access bajo la licencia CC BY-NC-ND (http://creativecommons.org/licenses/by-nc-nd/4.o/). 
Resultados: Se realizó la identificación inicial de 62 artículos, se aplicaron los criterios de exclusión hasta el establecimiento de la muestra de 26 artículos. Después de la lectura y análisis de los estudios, se constató que las tecnologías son más utilizadas junto con las clases teóricas en las aulas, o como plataformas online de enseñanza, o también como herramientas auxiliares para la práctica de simulaciones realísticas.

Discusión: El uso de internet fue predominante en los estudios, pues favorece la actualización, la comunicación y la cualificación académica. En cuanto a las plataformas digitales de enseñanza, el espacio virtual permite reunir e integrar diversos medios con finalidades diferentes posibilitando la creación de actividades variadas. Para la enseñanza a través de simulaciones se presentaron diversos beneficios de inmersión a un ambiente profesional desde la universidad.

Conclusiones: El estudio presenta una visión general de las tecnologías educativas utilizadas para la enseñanza de enfermería y provee conocimiento de nuevas metodologías, lo que beneficia a profesores y estudiantes.

Palabras clave: Tecnología educativa; educación en enfermería; plataformas virtuales; simulación clínica; Brasil.

\begin{abstract}
Introduction: The utilization of modern digital resources in teaching constitutes a strategy to address the new learning needs. In the area of nursing, the use of digital resources can facilitate the insertion of students into the clinical practice by providing a virtual environment in which students can develop their skills and competencies.

Objective: To analyze the evidence regarding the utilization of technologies in nursing teaching.

Methods: This is an integrative review based on the SciELO publications and IBECS, LILACS, BDENF, Web of Science, PubMed and CINAHL databases.

Results: 62 relevant articles were identified. After considering the exclusion criteria, a final sample with 26 articles was constituted. The studies were read and analyzed, and it was found that the diverse technologies are more frequently utilized in conjunction with theory classes in classrooms, as online teaching platforms, or as auxiliary tools to support realistic simulations.

Discussion: The use of the Internet was prevalent in the analyzed studies. Regarding some teaching digital platforms, virtual spaces showed being useful to create diverse teaching-learning activities; with simulations being helpful to prepare students to enter the professional practice.

Conclusions: This study presents a general view of the educational technologies utilized in nursing teaching, while displaying some specific methodologies which can be of benefit for both students and teachers.

Keywords: Educational technology; nursing education; virtual platforms; clinical simulation; Brazil.
\end{abstract}

\title{
RESUMO
}

Introdução: A utilização de recursos digitais modernos para o ensino constitui uma estratégia para atender as novas necessidades de aprendizagem. No âmbito de enfermagem 
pode facilitar a inserção dos estudantes na prática clínica em modalidade virtual para que desenvolvam suas habilidades e competências.

Objetivo: Analisar as evidências sobre a utilização de tecnologias no ensino de enfermagem. Métodos: Revisão integrativa realizada no portal de periódicos SciELO e nas bases de dados IBECS, LILACS, BDENF, Web of Science, PubMed e CINAHL.

Resultados: Foi realizada a identificação inicial de 62 artigos, aplicaram-se os critérios de exclusão até o estabelecimento da amostra de 26 artigos. Após a leitura e a análise dos estudos, foi constatado que as tecnologias são mais utilizadas em conjunto com as aulas teóricas nas salas de aula, ou como plataformas de ensino online, ou também como ferramentas auxiliares para a prática de simulações realísticas.

Discussão: O uso da internet foi predominante nos estudos, dado que favorece a atualização, a comunicação e a qualificação acadêmica. Sobre as plataformas digitais de ensino, o espaço virtual permite reunir e integrar diversos médios com finalidades diferentes possibilitando a criação de atividades variadas. Apresentaram-se diversos benefícios de imersão para o ensino através de simulações em um ambiente profissional desde a universidade.

Conclusões: O estudo apresenta uma visão geral das tecnologias educacionais utilizadas no ensino de enfermagem e provê conhecimento de novas metodologias, o qual beneficia professores e estudantes.

Palavras chave: Tecnologia educacional; educação em enfermagem; plataformas virtuais; simulação clínica; Brasil.

\section{INTRODUCCIÓN}

Hoy en día, la población está viviendo una nueva revolución conocida como era digital. Sus impactos han causado una modificación significativa en la velocidad de producción y diseminación de las informaciones, así como en el desarrollo tecnológico. Los estudios sobre las tecnologías digitales crecen día con día, al igual que las investigaciones que permiten conocer cómo utilizarlas para vivir mejor, producir más, relacionarse, y sobre todo, aprender y enseñar de forma más eficiente y adecuada ${ }^{1}$.

En ese contexto también se observa la utilización de las tecnologías en el ámbito de la enseñanza. Con la evolución de las tecnologías digitales, un paradigma diferente se construye en relación al proceso de enseñanza y aprendizaje, con cambios necesarios en el contexto educacional actual. Las tecnologías representan una oportunidad para propiciar cambios significativos en la educación, especialmente en relación a la práctica docente centrada en el profesor para centrarla en los alumnos, como forma de corresponder a sus demandas de conocimiento².

La utilización de tecnologías en el ámbito de enseñanza de la enfermería se ha vuelto cada vez más común, pues se busca promover una enseñanza innovadora y dinámica de los académicos. Ante esto, es importante destacar que la formación de enfermería ha sufrido diversos cambios, tales como: el reconocimiento del carácter multidisciplinario de la práctica profesional, el estímulo al raciocinio clínico, la valorización de la articulación teórica y práctica, la utilización de metodologías activas de enseñanza/aprendizaje, además de la flexibilidad curricular³.

Se entiende como tecnologías para la enseñanza la inserción de herramientas tecnológicas con fines pedagógicos en ambientes de aprendizaje, teniendo presente que, en la enfermería, la 
relación docente-estudiante rebasa el panorama académico, como también ocurre en la relación enfermero-paciente, y en el proceso de trabajo en salud3.

La utilización de las tecnologías educacionales para alumnos de enfermería proporciona una mayor capacitación de sus habilidades prácticas4 ${ }^{4}$. El uso de los recursos digitales en la enseñanza propicia una mayor dinamización en el método tradicional del aula y se favorece el interés de los alumnos. Con la aproximación a una situación real a partir de estos recursos, es posible, además, optimizar su tiempo de estudio y obtener la percepción de la responsabilidad por el propio aprendizajes.

Asimismo, el uso de tecnologías puede facilitar la inserción de los estudiantes en la práctica clínica, aunque de modo virtual, para que desarrollen sus habilidades y competencias dentro del contexto de enseñanza, con menor probabilidad de errores o daños, lo que determina la calidad del cuidado en salud.

Dados los beneficios del uso de tecnologías para la enseñanza, la realización de una revisión integrativa posibilita la búsqueda, síntesis y análisis sistematizado de la investigación ya desarrollada sobre el tema. Aunado a esto, se contribuye a la profundización y difusión de los principales resultados relacionados con el uso de tecnologías en el contexto de la educación en enfermería.

Con base en lo anterior, es necesario investigar qué estrategias modernas de enseñanza se pueden utilizar para capacitar a enfermeras(os), profesionales con gran responsabilidad en el cuidado de otros en el campo de la salud, con el fin de ofrecer una atención segura y de calidad.

Teniendo en cuenta los beneficios del uso de la tecnología dentro de la enseñanza de enfermería y su relevancia para la seguridad del paciente, se estableció como objetivo analizar las evidencias sobre la utilización de tecnologías en la enseñanza de enfermería.

\section{MÉTODOS}

Se trata de una revisión integrativa de la literatura que siguió las siguientes etapas: 1. Identificación del tema y selección de la cuestión de la investigación; 2. Establecimiento de criterios para inclusión y exclusión de estudios; 3. Categorización de los estudios; 4. Evaluación de los estudios incluidos en la revisión integrativa; 5 . Interpretación de los resultados y 6. Síntesis del conocimiento ${ }^{6}$.

La pregunta guía de la investigación fue elaborada a partir de la estrategia PICO: ¿Cuál es la producción científica reciente sobre las tecnologías utilizadas en la enseñanza de enfermería?

Se utilizaron como fuentes el portal de revistas Scientific Electronic Library Online (SciELO) y las bases de datos Índice Bibliográfico Español en Ciencias de la Salud (IBECS), Literatura LatinoAmericana y del Caribe en Ciencias de la Salud (LILACS), Base de datos de enfermería (BDENF), Web of Science (WOS), Library of Medicine National Institutes of Health (PubMed), Cumulative Index to Nursing and Allied Health Literature (CINAHL).

La búsqueda fue realizada de enero a marzo de 2019, se utilizaron los descriptores controlados DeCS "Education, Nursing, Baccalaureate" AND "Educational Technology" AND "Education, Nursing" (con sus variantes en portugués y español) para el portal de revistas SciELO y bases IBESCS, LILACS y BDENF, así como los descriptores MeSH "Education, Nursing, Baccalaureate" OR "Education, Nursing, Graduate" AND "Educational Technology" AND "Education, Nursing" en las bases WOS, PubMed y CINAHL.

Se establecieron como criterios de inclusión estudios primarios publicados en el período de 2009 a 2019, en los idiomas portugués, inglés y español, y como criterios de exclusión revisiones de literatura, capítulos, cartas, editoriales, relatos de experiencia y estudios de caso. 
La selección de estudios primarios fue realizada por pares; para el análisis de la información de los estudios se utilizó un instrumento adaptado por Michelato et al.7, el cual contempla los ítems: autoría del estudio, año y país de publicación, características metodológicas, evaluación del nivel de evidencia y tecnología de enseñanza abordada.

Se analizó el nivel de evidencia a partir de la jerarquía en siete niveles de Mazurek y FineoutOverholt ${ }^{8}$ : Nivel 1) evidencias provenientes de revisión sistemática o meta-análisis de ensayos clínicos aleatorizados controlados; Nivel 2) evidencias derivadas de ensayo clínico aleatorizado controlado; Nivel 3) evidencias de ensayos clínicos sin aleatorización; Nivel 4) evidencias obtenidas de estudios de cohorte o de caso control; Nivel 5) evidencias de meta-síntesis de estudios descriptivos o cualitativos; Nivel 6) evidencias originadas de un único estudio descriptivo o cualitativo; Nivel 7) evidencias de opiniones de expertos o autoridades.

Finalmente, se adoptó el análisis descriptivo para conocer las tecnologías desarrolladas para la enseñanza de enfermería, así como las ventajas y desventajas de su utilización.

\section{RESULTADOS}

En las búsquedas realizadas en las bases de datos seleccionadas y el portal de revistas fueron identificados, inicialmente, 63 artículos relacionados con los descriptores especificados y criterios deinclusión.Sefueronexcluyendoartículos conforme alos criterios establecidos metodológicamente, a partir de la lectura de títulos, resúmenes y trabajos completos, hasta el establecimiento de la muestra final de 26 artículos. El proceso de selección fue realizado conforme al diagrama de flujo ilustrado en la Figura 1.

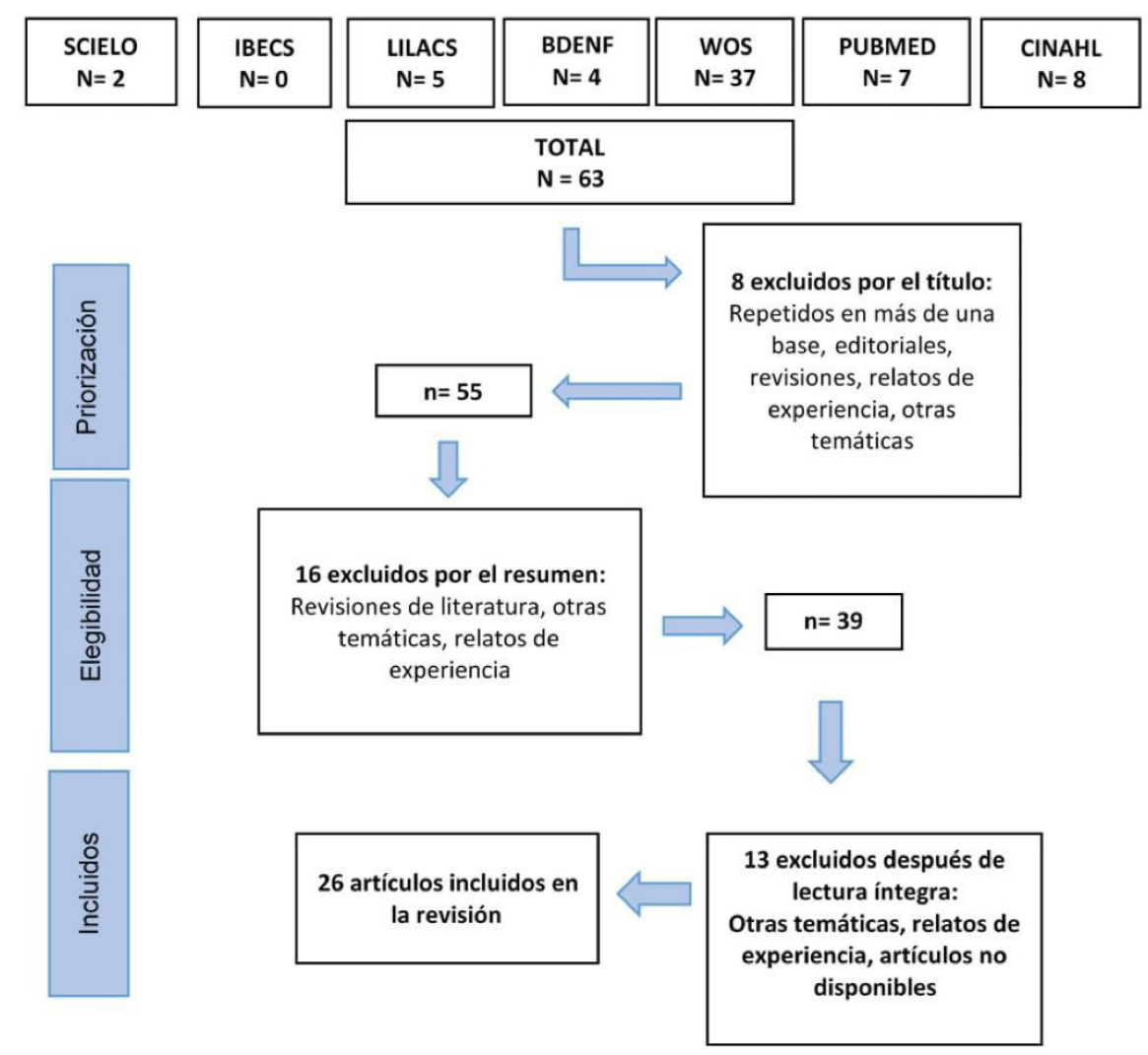

Figura 1. Diagrama de selección de los estudios adaptado del Preferred Reporting Items for Systematic Reviews and Meta-Analyses (PRISMA) 
Con relación a la caracterización de los estudios seleccionados, se observó que la publicación sobre la utilización de tecnologías en la enseñanza de enfermería es reciente, ya que la mayoría de los artículos se publicaron en años posteriores a 2015.

En cuanto al país de origen, hubo predominancia de estudios provenientes de los Estados Unidos (14 publicaciones), seguido por Brasil (5 estudios). Sin embargo, se observa que diversas naciones en el mundo están buscando invertir en tecnología educacional, ya que otros países también fueron identificados, tales como: Australia, Suecia, China, Nueva Zelanda, Corea del Sur y Reino Unido.

Concerniente al tipo de estudio y nivel de evidencia, prevalecieron los estudios descriptivos, seguidos por los cuasi-experimentales y los metodológicos que describían el desarrollo de las tecnologías educacionales. Sólo un estudio realizado en Corea del Sur tuvo mayor nivel de evidencia (II). A continuación, se presenta la síntesis de los artículos seleccionados con la caracterización en el Cuadro 1.

\begin{tabular}{lcccc}
\multicolumn{2}{c}{ Cuadro 1. Caracteristicas de los estudios primarios seleccionados } \\
para la revisión integradora. Fortaleza-CE, 2018
\end{tabular}




\begin{tabular}{|c|c|c|c|c|}
\hline Carruth AK. et al. ${ }^{19}$ & 2010/EUA & $\begin{array}{l}\text { Descriptivo, } \\
\text { cualitativo }\end{array}$ & VI & $\begin{array}{l}\text { E-learning para simulación de } \\
\text { actividades del v de enfermería. }\end{array}$ \\
\hline Beard K, Morote ES. ${ }^{20}$ & 2010/EUA & $\begin{array}{l}\text { Cuasi- } \\
\text { experimental }\end{array}$ & III & $\begin{array}{l}\text { Audios en podcasts como } \\
\text { instrumento de enseñanza basado en } \\
\text { la pedagogía narrativa. }\end{array}$ \\
\hline $\begin{array}{l}\text { Kardong-Edgren S, } \\
\text { Emerson R. } .^{21}\end{array}$ & 2010/EUA & $\begin{array}{l}\text { Descriptivo, } \\
\text { cualitativo }\end{array}$ & $\mathrm{VI}$ & $\begin{array}{l}\text { Aulas y audios en podcasts en el } \\
\text { curso de enfermería. }\end{array}$ \\
\hline Godbold R, Lees A. ${ }^{22}$ & $\begin{array}{l}\text { 2016/ } \\
\text { Nueva } \\
\text { Zelanda }\end{array}$ & $\begin{array}{l}\text { evaluación de } \\
\text { tecnología, } \\
\text { cualitativo }\end{array}$ & VI & $\begin{array}{l}\text { Espacio online exclusivo (Values } \\
\text { Exchange) como ambiente de pre- } \\
\text { registro seguro para la deliberación } \\
\text { de casos. }\end{array}$ \\
\hline Fiedler R. et al. ${ }^{23}$ & 2014/EUA & $\begin{array}{l}\text { Descriptivo, } \\
\text { cualitativo }\end{array}$ & VI & $\begin{array}{c}\text { Tecnología The Neighborhood } \\
\text { (comunidad virtual) para compromiso } \\
\text { de los alumnos. }\end{array}$ \\
\hline Alvarez AG. et al. ${ }^{24}$ & $\begin{array}{l}2017 / \\
\text { Brasil }\end{array}$ & $\begin{array}{l}\text { Cuasi- } \\
\text { experimental }\end{array}$ & III & $\begin{array}{l}\text { Intervención educacional online para } \\
\text { evaluación del dolor agudo. }\end{array}$ \\
\hline Guhde J. ${ }^{25}$ & 2010/EUA & $\begin{array}{l}\text { Descriptivo, } \\
\text { cualitativo }\end{array}$ & VI & $\begin{array}{l}\text { Videos y discusiones online para } \\
\text { estimular el pensamiento crítico en } \\
\text { estudiantes de enfermería }\end{array}$ \\
\hline $\begin{array}{l}\text { Costa-Valcanti Avelino } \\
\text { C. et al. }{ }^{26}\end{array}$ & $\begin{array}{l}2017 / \\
\text { Brasil }\end{array}$ & $\begin{array}{l}\text { Descriptivo, } \\
\text { cualitativo }\end{array}$ & $\mathrm{VI}$ & $\begin{array}{l}\text { Curso en la plataforma Moodle } \\
\text { para enseñanza de la Clasificación } \\
\text { Internacional para la Práctica de } \\
\text { Enfermería (CIPE). }\end{array}$ \\
\hline Tibes CM. et al. ${ }^{27}$ & $\begin{array}{l}2017 / \\
\text { Brasil }\end{array}$ & Descriptivo & VI & $\begin{array}{c}\text { Tecnología WebQuest para auxiliar } \\
\text { en el proceso de enseñanza y } \\
\text { aprendizaje de estudiantes de } \\
\text { enfermería. }\end{array}$ \\
\hline Lee NJ. et al. ${ }^{28}$ & $\begin{array}{l}\text { 2016/ } \\
\text { Corea del Sur }\end{array}$ & $\begin{array}{l}\text { Ensayo clínico } \\
\text { aleatorizado, } \\
\text { controlado. }\end{array}$ & II & $\begin{array}{l}\text { Videoclip de motivación del } \\
\text { aprendizaje de cateterismo urinario. }\end{array}$ \\
\hline Mennenga HA. et al. ${ }^{29}$ & 2016/EUA & $\begin{array}{l}\text { Cuasi- } \\
\text { experimental }\end{array}$ & III & Libros didácticos digitales. \\
\hline Power T. et al. ${ }^{30}$ & $\begin{array}{c}\text { 2015/ } \\
\text { Australia }\end{array}$ & De cohorte & IV & $\begin{array}{l}\text { Utilización de maniquíes y casos } \\
\text { clínicos para la práctica de simulación. }\end{array}$ \\
\hline Au ML. et al. ${ }^{31}$ & $\begin{array}{l}2016 / \\
\text { China }\end{array}$ & $\begin{array}{l}\text { Descriptivo, } \\
\text { cualitativo }\end{array}$ & $\mathrm{VI}$ & $\begin{array}{l}\text { Simulación de alta fidelidad en } \\
\text { enseñanza de enfermería }\end{array}$ \\
\hline $\begin{array}{l}\text { Shinnick MA, Woo } \\
\text { MA. }^{32}\end{array}$ & 2015/EUA & Cohorte & IV & $\begin{array}{c}\text { Simulación de pacientes humanos } \\
\text { con insuficiencia cardíaca para la } \\
\text { enseñanza de enfermería. }\end{array}$ \\
\hline Marken PA. et al. ${ }^{33}$ & 2010/EUA & $\begin{array}{c}\text { Descriptivo, } \\
\text { cualitativo }\end{array}$ & VI & $\begin{array}{l}\text { Simulación para la enseñanza de } \\
\text { equipos multi-profesionales sobre } \\
\text { temas difíciles con los pacientes. }\end{array}$ \\
\hline Nyström S. et al. ${ }^{34}$ & $\begin{array}{l}\text { 2016/ } \\
\text { Suecia }\end{array}$ & $\begin{array}{l}\text { Cuasi- } \\
\text { experimental }\end{array}$ & III & $\begin{array}{l}\text { Debriefing después de simulación } \\
\text { como práctica de apoyo a los alumnos } \\
\text { en el aprendizaje interprofesional. }\end{array}$ \\
\hline
\end{tabular}

Después de la lectura completa de los artículos seleccionados se optó por agrupar las tecnologías de enseñanza utilizadas en los cursos de enfermería en tres ejes para describirlos mejor: a) Tecnologías desarrolladas para uso en aulas presenciales, b) Plataformas online para enseñanza a distancia, y c) Nuevos abordajes para la práctica de simulaciones realísticas. 


\section{a) Tecnologías desarrolladas para uso en aulas presenciales}

Se constató que gran parte de los estudios mostró experiencias en las cuales la tecnología es utilizada en asociación a las prácticas tradicionales de enseñanza, como forma de buscar abordajes metodológicos innovadores. Los autores afirman que los estudiantes modernos constituyen un desafío único para los educadores, exigiendo al profesor el empleo constante de nuevas estrategias de enseñanza. Este compromiso activo aumenta la capacidad de respuesta y fomenta la participación de los estudiantes en las clases9.

Cabe señalar que los resultados fueron analizados con el fin de identificar cuáles tecnologías consiguieron ser bien insertadas en la enseñanza de enfermería, así como los impactos en el aprendizaje de los estudiantes.

En Australia se utilizaron asistentes personales digitales durante una clase de clínica médicoquirúrgica de un curso de bachillerato en enfermería. Los resultados mostraron que los estudiantes que usaron la tecnología tuvieron un aumento en el puntaje promedio acerca del conocimiento farmacológico y clínico, con el doble de aumento en el grupo control ${ }^{10}$.

En otro estudio desarrollado en los Estados Unidos, los autores buscaron evaluar la satisfacción de los alumnos para una estrategia de enseñanza de multimedia proyectada por la facultad y encontraron que el uso de la tecnología en la sala de aula involucró a los alumnos en el proceso de aprendizaje y facilitó la exposición de contenidos más difíciles. Los estudiantes expresaron preferencia por todos los materiales de instrucción presentados en un formato animado ${ }^{11}$.

Otra investigación realizada también en Estados Unidos abordó la utilización del Google Glass $^{\circ}$ como herramienta de inmersión en un ambiente de simulación de alta fidelidad, respecto a la seguridad para preparar medicamentos. Se identificó que la utilización de la simulación de alta fidelidad propició comportamientos positivos y competencias para preparar medicamentos ${ }^{12}$.

También, en algunos estudios ${ }^{13-15}$ destacó el uso de la tecnología denominada clickers, es decir, dispositivos de control remoto de bolsillo que usan señales para transmitir y grabar las respuestas de preguntas realizadas durante el aula.

En general, los hallazgos demostraron que los alumnos estaban satisfechos con el uso de los clickers en el aula y que la tecnología puede ayudar en la adquisición de conocimiento y retención de contenido ${ }^{14}$. Además, se constató que los estudiantes percibieron los clickers como una adición positiva para el ambiente en el aula, pareciendo aumentar la interacción y compromiso ${ }^{13}$. Sin embargo, en uno de los artículos los resultados del uso de clickers para la enseñanza de la farmacología en enfermería no parecía mejorar la comprensión o la retención de los estudiantes en comparación con los que no los utilizaban ${ }^{15}$, demostrando que la tecnología no siempre se adapta a todas las realidades de la enseñanza.

La introducción de tecnología móvil a través de iPads se describió en un estudio en Nueva Zelanda que tenía como objetivo apoyar el aprendizaje de los alumnos en ambientes clínicos. Los resultados presentaron factores positivos y negativos. La participación de los estudiantes y la conectividad fueron elementos facilitadores del aprendizaje y la principal barrera fue la baja experiencia del profesorado, revelando que los profesores aún necesitan adaptarse a las nuevas metodologías de aprendizaje ${ }^{16}$.

El uso de tecnologías también fue insertado para las evaluaciones académicas en una investigación desarrollada en el Reino Unido. En el proceso de transición de documentos de evaluación impresos hacia electrónicos, se obtuvo que con entrenamiento apropiado y apoyo y pese a algunas ansiedades, los estudiantes y sus profesores pueden desarrollar las habilidades necesarias para usar con éxito una herramienta digital ${ }^{17}$. 


\section{b) Plataformas online para enseñanza a distancia}

Con la diseminación global de internet y la creciente tendencia de los abordajes de enseñanza a distancia, como complemento de las aulas presenciales, algunos estudios obtuvieron resultados positivos de herramientas tecnológicas utilizadas en cursos de enfermería.

Un estudio brasileño destacó el uso de internet como herramienta complementaria en el proceso de enseñanza-aprendizaje, demostrando la importancia de este dispositivo para el crecimiento cognitivo ${ }^{18}$.

En una investigación realizada en Estados Unidos los autores describieron la utilización de un curso de orientación online con actividades virtuales simuladas, esto proporcionó a los estudiantes una innovación que garantizó una experiencia virtual positiva del aprendizaje. De igual forma, el estudio mostró un cambio de actividades de e-learning y su utilización promovió un ambiente de aprendizaje saludable para los profesores y académicos ${ }^{19}$.

A su vez, también fue importante la utilización de foros podcasted en cursos de graduación en enfermería. Se percibió que, inicialmente, a la mayoría de los estudiantes no les era familiar el uso de podcasts en la educación y que después de familiarizarse con el formato, éstos relataron que los podcasts los ayudaron a estudiar y mejoraron sus notas. Asimismo, algunos resultados mostraron que la pedagogía narrativa es un método de enseñanza que puede ayudar a los estudiantes a alcanzar los objetivos de aprendizaje, además de la facilidad que, a través de la tecnología, puede ser implementada en conferencias virtuales ${ }^{20}$.

Otra investigación también describió el uso de conferencias en formato podcast en un curso de enfermería. Los alumnos, a pesar de no estar familiarizados inicialmente con el formato de las clases, se adaptaron bien a la tecnología que les ayudó a actualizar los contenidos²1.

Las tecnologías basadas en la Web también fueron descritas en algunos artículos. En Nueva Zelanda, el resultado fue la creación de una comunidad basada en la red que apoya usuarios de diversas disciplinas para pensar profundamente sobre cuestiones éticas de su práctica profesional. Esa tecnología proporcionó un espacio exclusivo a los estudiantes para integrar evidencias y valores en su toma de decisión, oportunidades auténticas para explorar la complejidad de tomar decisiones basadas en la práctica y promover la reflexión sobre cómo éstas impactan en la forma en que se brinda atención individual y colectiva ${ }^{22}$. Los profesores también relataron su aceptación y apoyo a la innovación en enseñanza después del uso de una comunidad virtual, la cual mejoró sus experiencias de enseñanza ${ }^{23}$.

Un estudio brasileño utilizó otra herramienta de enseñanza online: un objeto de aprendizaje virtual para la evaluación del dolor agudo. Se comprobó que el uso de la tecnología persuasiva en el aprendizaje de los estudiantes de enfermería puede ampliar los espacios de aprendizaje de una manera innovadora, flexible, motivadora y prometedora ${ }^{24}$

Los vídeos disponibles para el debate online también se describieron como una herramienta de aprendizaje en un artículo que pretendía utilizarlos para estimular la evaluación inicial del paciente y el pensamiento crítico con estudiantes de enfermería de nivel inicial. Utilizando la tecnología, los profesores observaron una mejora en la evaluación clínica del paciente y una mayor conciencia de la importancia de la anamnesis ${ }^{25}$.

En otro estudio brasileño fue desarrollado en ambiente virtual de aprendizaje un curso sobre diagnósticos, intervenciones y resultados de enfermería, utilizando la CIPE $^{\circ}$ (Clasificación Internacional para la Práctica de Enfermería ${ }^{26}$. De igual forma, se llevó a cabo la descripción de una tecnología denominada WebQuest, basada en la práctica de la investigación orientada por 
medio de un problema o desafío, con el fin de auxiliar en el proceso de enseñanza y aprendizaje de estudiantes y profesionales de enfermería ${ }^{27}$.

El empleo de dispositivos móviles también se destacó en los estudios. El uso de un videoclip para dispositivo móvil fue evaluado por los estudiantes para la educación y el desarrollo de habilidades de enfermería sobre cateterismo urinario. Según estos estudiantes, las ventajas de la educación basada en dispositivos móviles eran su accesibilidad sin restricciones de lugar y tiempo, disponibilidad para la preparación y revisión ${ }^{28}$.

A partir de estos estudios se constató que la utilización de nuevas tecnologías educacionales fue una estrategia innovadora que caracteriza un cambio de paradigma en la capacitación de estudiantes del área de la salud y contribuye positivamente para el aprendizaje, además de incentivar la participación de los alumnos, estimulada por los recursos tecnológicos.

\section{c) Nuevos abordajes para la práctica de simulaciones realistas}

Con base en la búsqueda, en el ámbito de enseñanza se destacó la utilización de simulaciones realistas como forma de integrar los recursos tecnológicos actuales a las clases teórico-prácticas de enfermería. Además, los autores creen que el uso de casos clínicos y simulaciones puede ayudar al desarrollo de la empatía de los estudiantes, que es fundamental para la relación enfermero-paciente ${ }^{29}$.

En Australia, una investigación describió las perspectivas de los alumnos de un enfoque mejorado en tecnología en capas para mejorar la simulación como experiencia de aprendizaje. Se trató de la introducción de estudios de caso a los alumnos a través de pegatinas para luego tener el contacto con los maniquíes a través de la simulación. Los resultados mostraron que los alumnos superaron el contacto con el plástico sabiendo qué decir; cuidando y conversando; y abrazando la diversidad del paciente. Los comentarios indicaron que esas medidas aumentaron la habilidad de los alumnos de suspender la incredulidad, sentirse conectado y aproximarse a los maniquíes de una forma más comprensiva y empática ${ }^{30}$.

Un estudio realizado en China con el objetivo de explorar la percepción de los estudiantes de enfermería sobre el uso de la simulación de alta fidelidad constató que las actividades de simulación fueron apreciadas por los estudiantes y contribuyeron principalmente para la habilidad técnica en la visión de los alumnos. Con todo, los profesores identificaron una deficiencia en el método al describir que algunos estudiantes actúan con desprecio hacia la vida del paciente simulado ${ }^{31}$.

En los Estados Unidos, un estudio que utilizó la simulación de pacientes humanos demostró que los profesores pueden usarla con confianza como un método de enseñanza auxiliar con el fin de que los estudiantes combinen la teoría y la práctica, ya que se obtendrán ganancias de conocimiento a partir de diferentes estilos de aprendizaje ${ }^{32}$.

Otro enfoque de la simulación fue su uso como herramienta para enseñar a los equipos interprofesionales cómo reconocer y entablar conversaciones difíciles con los pacientes sobre la violencia. Los estudiantes que participaron en el estudio se mostraron satisfechos con el uso de la simulación y afirmaron haber aprendido muchas lecciones valiosas a través del debriefing y de la autorreflexión ${ }^{33}$.

En las simulaciones se destacó la práctica del debriefing como parte imprescindible de evaluación del proceso de aprendizaje. En una investigación sueca, su utilización fue integrada a las actividades de simulación con estudiantes de medicina y enfermería como instrumento de enseñanza interprofesional. Los resultados fueron sorprendentes y revelaron que la estructura 
impuesta del debriefing no aseguró la colaboración interprofesional, hecho que emerge como un tema importante para la reflexión respecto a la formación de los profesionales de salud ${ }^{34}$, pues se señala que la imposición de la simulación no garantiza el aprendizaje e interacción entre los alumnos.

Los resultados de los estudios de este eje revelaron fragilidades en la utilización de las simulaciones realísticas. Ante esto, es fundamental destacar que la tecnología por sí sola no garantiza el aprendizaje, por lo tanto, los profesores deben utilizarla con dominio y cautela.

\section{DISCUSIÓN}

Con la revisión de estas experiencias relatadas en la literatura, se constató que la utilización de la internet fue predominante y que los estudiantes y profesores de enfermería consideran su utilización como una herramienta complementaria al proceso enseñanza-aprendizaje, pues favorece la actualización, la comunicación y la cualificación académica, con impacto positivo en el crecimiento científico35.

También se confirma la disponibilidad de una amplia variedad de recursos digitales que pueden apoyar y favorecer la educación de enfermería, como el sistema CIPE, la WebQuest, Google Glass ${ }^{\circledR}$, el uso de clickers y podcasts. En lo que respecta al uso de plataformas digitales, un estudio brasileño destaca que la enfermería es considerada por muchos una profesión esencialmente práctica. Sin embargo, este hecho no se opone a la utilización de enseñanza a la distancia como forma de viabilizar la educación continua a través de la realización de cursos de extensión y posgraduación online, ya que el espacio virtual permite reunir e integrar diversos medios con finalidades diferentes, además posibilita la creación de actividades variadas y multifacéticas ${ }^{36}$. Se acepta que la utilización de nuevas tecnologías online en la enseñanza de enfermería proporciona al alumno una nueva forma de aprender, puesto que utiliza estrategias educacionales que facilitan el aprendizaje en una perspectiva interactiva, también le ofrece oportunidades de nuevas experiencias a través de la enseñanza en la modalidad a distancia4.

En la utilización de simulaciones, se destacaron los diversos beneficios de inmersión en un ambiente profesional desde la universidad. En ese contexto, los autores afirman en sus investigaciones que la utilización de los simuladores posibilita la presentación de diversos escenarios dentro de un ambiente controlado. Otro aspecto relevante de la simulación es que el aprendizaje realizado en la situación planeada permite la apropiación de los conocimientos y habilidades necesarias de forma rápida y eficiente ${ }^{37}$.

Aunado a lo anterior, un beneficio del uso de las simulaciones se observa en la evaluación de los alumnos, donde es posible analizar el desarrollo de habilidades de procedimientos y de pensamiento crítico, lo que se refleja con mejor calidad del cuidado al paciente ${ }^{38}$.

En cuanto a la promoción de la seguridad del paciente, la utilización de simulaciones realistas en educación de los profesionales de salud contempla la práctica en un ambiente que permite errores y crecimiento profesional, sin colocar en riesgo al paciente. Por lo tanto, en situaciones programadas en escenarios protegidos y controlados es posible mejorar habilidades manuales y de relación durante el proceso de aprendizaje sin causar daños al paciente ${ }^{39}$. Además, el último paso de la simulación (conocido como debriefing) permite una discusión reflexiva de la situación y promueve el aprendizaje por medio del análisis de las decisiones tomadas, estimulando el pensamiento crítico y reflexivo del estudiante ${ }^{40}$. 
De igual forma, con el desarrollo de esa revisión fue percibido que el uso de simulaciones trae múltiples beneficios, aunquetambién presenta fragilidades, tales como no garantizar lainteraccióny comunicación profesional, así como la humanización en los cuidados. Este hecho despierta la necesidad de los profesores como mediadores de las situaciones simuladas, para obtener el mayor provecho de la tecnología educacional.

Con base en lo expuesto en los resultados del análisis, se percibió que los avances tecnológicos modernos son fuertes aliados para el desarrollo de metodologías para la enseñanza en la salud y proporcionan numerosos beneficios, siempre y cuando sean utilizados de forma consciente y supervisados por profesores capacitados.

\section{CONCLUSIONES}

El estudio proporcionó una visión global de la utilización de las tecnologías educacionales, con sus beneficios y fragilidades. Se destacó la contribución positiva para el proceso enseñanza-aprendizaje, ya que promueven la interacción, actualización y la cualificación académica. Sin embargo, las tecnologías deben ser utilizadas como herramienta auxiliar, pues individualmente no garantizan la enseñanza de atributos fundamentales al cuidado, tales como ética profesional y trabajo en equipo.

Las principales tecnologías utilizadas fueron las plataformas virtuales online, las simulaciones realistas, los videos educacionales, los libros digitales, los clickers y los podcasts. De ese modo, nuevas investigaciones de desarrollo de metodologías de enseñanza podrán ser realizadas basadas en las lagunas existentes en la literatura.

Por lo tanto, el estudio contribuye a la difusión y el fortalecimiento de estrategias actuales e innovadoras que pueden utilizarse para la formación de profesionales de enfermería, con una alta capacidad de pensamiento crítico-reflexivo, con el fin de ofrecer una atención segura y de calidad.

\section{RESPONSABILIDADES ÉTICAS}

Experimentos con personas o animales. No procede, esta fue una investigación documental.

Conflicto de intereses. Los autores declaran no tener conflicto de intereses.

Financiamiento. Ninguno.

\section{REFERENCIAS}

1. Gabriel M. Educ@r-A revolução digital na educação. São Paulo: Saraiva; 2013.

2. De Oliveira-Costa RR, De Medeiros SM, Martins JCA, Paiva-de Menezes RM, Souto-de Araújo M. O uso da simulação no contexto da educação e formação em saúde e enfermagem: uma reflexão acadêmica. Espaço para a saúde. 2016; 16(1): 59-65. https://n9.cl/zo6wr

3. Candido-de Oliveira Salvador PT, Filgueira-Martins CC, Andrade-Alves KY, Silvanêre-Pereira M, Pereira-Santos VE, Solange-Vieira-Tourinho F. Tecnologia no ensino de enfermagem. Rev. Baiana Enferm. 2015; 29(1): 34-4. https://ng.cl/lbbf

4. Marques-Frota N, Moreira-Barros L, Moura-de Araújo T, Nunes-Caldini L, Cândido-do Nascimento J, Áfio-Caetano J. Construção de uma tecnologia educacional para o ensino de enfermagem sobre punção venosa periférica. Rev. gaúcha enferm. 2013; 34(2): 29-36. https://bit.ly/3bOQ_Wf

5. 5. Tanaka RY, Menezes-Catala V, Zemiack J, Rubim-Pedro EN, Petersen-Cogo AL, Tolfo-Silveira D. Objeto educacional digital: avaliação da ferramenta para prática de ensino em enfermagem. Acta Paul Enferm. 2010; 23(5): 603-7. https://doi.org/10.1590/S0103-21002010000500003 
6. Dal Sasso-Mendes K, De Campos-Pereira Silveira RC, Galvão CM. Integrative literature review: a research method to incorporate evidence in health care and nursing. Texto contexto enferm. 2008; 17(4): 758-64. https://doi.org/10.1590/S0104-07072008000400018

7. Michelato-Silva N, Dos Santos MA, Rodrigues-Rosado S, Galvão CG, Megumi-Sonobe H. Psychological aspects of patients with intestinal stoma: integrative review. Rev. Latino-Am. Enfermagem. 2017; 25: 1-11. http://dx.doi.org/10.1590/1518-8345.2231.2950

8. Mazurek-Melnyk B, Fineout-Overholt E. Making the case for evidence-based practice and cultivating a spirit of inquiry. En:Melnyk BM, Fineout-Overholt E. Evidence-based practice in nursing \& healthcare. A guide to best practice. $2^{\text {nd }}$ ed. Philadelphia: Wolters Kluwer/Lippincott Williams \& Wilkins; 2011.

9. Montenery SM, Walker M, Sorensen E, Thompson R, Kirklin D, White R, et al. Millennial generation student nurses' perceptions of the impact of multiple technologies on learning. Nurs Educ Perspect. 2013; 34(6): 405-9. https://doi.org/10.5480/10-451

10. Farrell MJ, Rose L. Use of mobile handheld computers in clinical nursing education. J Nurs Educ. 2008; 47(1): 13-9. https://doi.org/10.3928/01484834-20080101-03

11. Wolf L, Rutar P, Delgado C, Niederriter J. The design process of a multimodal module that synthesized knowledge across nursing courses. Nurse Educ Today. 2017; 52: 40-2.

https://doi.org/10.1016/j.nedt.2017.02.010

12. Schneidereith $\mathrm{T}$. Seeing through google glass: using na innovative technology to improve medication safety behaviors in undergraduate nursing students. Nurs Educ Perspect. 2015; 36(5): 337-9.

https://doi.org/10.5480/15-1653

13. Patterson B, Kilpatrick J, Woebkenberg E. Evidence for teaching practice: the impact of clickers in a large classroom environment. Nurse Educ Today. 2010; 30(7): 603-7.

https://doi.org/10.1016/j.nedt.2009.12.008

14. Meedzan N, Fisher K. Clickers in nursing education: an active learning tool in the classroom. OJNI. 2009; 13(2): 1-19. https://bit.ly/3vmpueO

15. Vana KD, Silva GE, Muzkaya D, Hirani LM. Effectiveness of an audience response system in teaching pharmacology to baccalaureate nursing students. Comput Inform Nurs. 2011; 29(6): 326-34. https://doi.org/10.1097/NCN.obo13e3181fgddgc

16. Mackay BJ, Anderson J, Harding T. Mobile technology in clinical teaching. Nurse Educ Pract. 2017; 22:1-6. https://doi.org/10.1016/j.nepr.2016.11.001

17. Smith J, Cambers W. Using an electronic assessment system for nursing students on placements. BJN. 2017; 26(21): 603-7. https://doi.org/10.12968/bjon.2017.26.21.1192

18. Souza-Leite KN, Ribeiro-dos Santos S, Sgren-da Costa Andrade S, Lacet-Zaccara AA, Ferreira-da Costa T. A internet e sua influência no processo ensino aprendizagem de estudantes de enfermagem. Rev. enferm. UERJ. 2013; 21(4): 464-70. https://bit.ly/3bO_ExA

19. Carruth AK, Broussard PC, Waldmeier VP, Gauthier DM, Mixon G. Graduate nursing online orientation course: transitioning for success. J Nur Educ. 2010; 49(12): 687-90.

https://doi.org/10.3928/01484834-20100831-06

20. Beard K, Morote ES. Using podcasts with narrative pedagogy: are learning objectives met? Nurs Educ Perspect. 2010; 31(3): 186-7. https://bit.ly/3vuLJiU

21. Kardong-Edgren S, Emerson R. Student adoption and perception of lecture podcasts in undergraduate bachelor of science in nursing courses. J Nurs Educ. 2010; 49(7): 398-401.

https://doi.org/10.3928/01484834-20100224-04 
22. Godbold R, Lees A. Facilitating values awareness through the education of health professionals: can web based decision making technology help? Nurse Educ Pract. 2016; 17: 193-9.

https://doi.org/10.1016/j.nepr.2015.11.003

23. Fiedler R, Giddens J, North S. Faculty experience of a technological innovation in nursing education. Nurs Educ Perspect. 2014; 35(6): 387-91. https://doi.org/10.5480/13-1188

24. Alvarez AG, Marcon-Dal Sasso GT, Iyengar MS. Persuasive technology in teaching acute pain assessment in nursing: results in learning based on pre and post-testing. Nurse Educ Today. 2017; 50: 109-14. https://doi.org/10.1016/j.nedt.2016.12.019

25. Guhde J. Combining simulation, instructor-produced videos, and online discussions to stimulate critical thinking in nursing students. Cin-computers informatics nursing. 2010; 28(5): 274-9. Avaliable from: https://www.ncbi.nlm.nih.gov/pubmed/20736724.

26. Costa-Valcanti Avelino C, Scalon-da Costa LC, Marques-Buchhorn SM, Alves-Nogueira D, TakamatsuGoyatá SL. Avaliação do ensino-aprendizagem sobre a CIPE ${ }^{\circledR}$ utilizando o ambiente virtual de aprendizagem. Rev. Bras. Enferm. 2017; 70(3): 630-7. https://doi.org/10.1590/0034-7167-2016-0545

27. Tibes CM, Dias JD, Westin UM, Domingues AN, Zem-Mascarenhas SH, Martinez-Évora YD. Desenvolvimento de recursos educacionais digitais para o ensino em enfermagem. Rev. enferm. UFPE on line. 2017; 11(Supl. 3):1326-34. https://doi.org/10.5205/reuol.10263-91568-1-RV.1103sup201702

28. Lee NJ, Chae SM, Kim H, Lee JH, Min HJ, Park DE. Mobile-based video learning outcomes in clinical nursing skill education: a randomized controlled trial. 2016; 34(1): 8-16.

https://doi.org/10.1097/CIN.0000000000000183

29. Mennenga HA, Bassett S, Pasquariello L. Empathy development through case study and simulation. Nurse Educ. 2016; 41(3): 139-42. https://doi.org/10.1097/NNE.0000000000000226

30. Power T, Virdun C, White H, Hayes C, Parker N, Kelly M, et al. Plastic with personality: increasing student engagement with manikins. Nurse Educ Today. 2016; 38: 126-31.

https://doi.org/10.1016/j.nedt.2015.12.001

31. Au ML, Lo MS, Cheong W, Wang SC, Van IK. Nursing students' perception of high-fidelity simulation activity instead of clinical placement: a qualitative study. Nurse Educ Today. 2016; 39: 16-21. https://doi.org/10.1016/j.nedt.2016.01.015

32. Shinnick MA, Woo MA. Learning style impact on knowledge gains in human patient simulation. Nurse Educ Today. 2015; 35: 63-7. https://doi.org/10.1016/j.nedt.2014.05.013

33. Marken PA, Zimmerman C, Kennedy C, Schremmer R, Smith KV. Human simulators and standardized patients to teach difficult conversations to interprofessional health care teams. Am J Pharm Educ. 2010; 74(7): 1-8. https://doi.org/10.5688/aj7407120

34. Nyström S, Dahlberg J, Edelbring S, Hult H, Dahlgren MA. Debriefing practices in interprofissional simulation with students: a sociomaterial perspective. BMC Med Educ. 2016; 16: 1-8.

https://doi.org/10.1186/s12909-016-0666-5

35. Cardoso-Tavares AP, Leite BS, Andrade-Silveira I, Dias-dos Santos T, De Andrade-Pereira de Brito W, LeiteFunchal Camacho AC. Análise das publicações nacionais sobre educação a distância em enfermagem: revisão integrativa. Rev. Bras. Enferm. 2018; 71(1): 227-36.http://dx.doi.org/10.1590/0034-7167-2016-0454

36. De Cassia-Vieira Rodrigues R, Ciqueto-Peres HH. Panorama brasileiro do ensino de Enfermagem On-line. Rev. esc. enferm. 2008; 42(2): 298-394. https://doi.org/10.1590/Soo80-62342008000200013

37. Satoko-Mizoi C, Utiyama-Kaneko RM, Moreira-Filho CA. A simulação realística como estratégia de treinamento para profissionais da saúde. einstein: Educ Contin Saúde. 2007; 5(3 Pt 2):100-1. https://ng.cl/w4ing 
38. De Souza-Teixeira CR, Kusumota L, Titareli-Merizio Martins Braga F, Pirani-Gaioso V, Dos Santos CB, Libório-de Sousa e Silva V, et al. O uso de simulador no ensino de avaliação clínica em enfermagem. Texto contexto enferm. 2011; 20: 187-93. http://bit.ly/3cQ5xVx

39. Gomes-de Abreu A, De Freitas JS, Berte M, Persegona-Ogradowski KR, Nestor A. O uso da simulação realística como metodologia de ensino e aprendizagem para as equipes de enfermagem de um hospital infanto-juvenil: relato de experiência. Ciência \& Saúde. 2014; 7(3):162-6.

https://doi.org/10.15448/1983-652X.2014.3.17874

40. Pleis-Neves Ferreira R, Mota-Guedes H, Willya-Douglas Oliveira D, Luiz-de Miranda J. Simulação realística como método de ensino no aprendizado de estudantes da área da saúde. Rev. Enferm. Cent.-Oeste Min. 2018; 8(2508): 1-9. https://doi.org/10.19175/recom.v8io.2508 